\title{
A High-Efficiency Approach for the Synthesis of N235-Impregnated Resins and the Application in Enhanced Adsorption and Separation of Vanadium(V)
}

\author{
Bo Chen ${ }^{1}$, Shenxu Bao ${ }^{1,2,3, *}$, Yimin Zhang ${ }^{1,2,3,4}$ and Ruwei Zheng ${ }^{1}$ \\ 1 School of Resources and Environmental Engineering, Wuhan University of Technology, \\ Wuhan 430070, China; bochen2012@whut.edu.cn (B.C.); zym126135@126.com (Y.Z.); \\ zhruwei@126.com (R.Z.) \\ 2 Hubei Key Laboratory of Mineral Resources Processing and Environment, Wuhan University of Technology, \\ Wuhan 430070, China \\ 3 Hubei Collaborative Innovation Center for High Efficient Utilization of Vanadium Resources, \\ Wuhan University of Science and Technology, Wuhan 430081, China \\ 4 State Environmental Protection Key Laboratory of Mineral Metallurgical Resources Utilization and Pollution \\ Control, Wuhan University of Science and Technology, Wuhan 430081, China \\ * Correspondence: sxbao@whut.edu.cn; Tel.: +86-27-8721-2127
}

Received: 13 July 2018; Accepted: 15 August 2018; Published: 19 August 2018

\begin{abstract}
Trialkylamine (N235)-tributyl phosphate (TBP) impregnated resins (N-TIRs) were prepared, so as to evaluate the effects of the addition of TBP on the preparation and adsorption performance of N235-impregnated resins (NIRs). The results show that TBP can obviously increase the impregnation ratio and shorten the impregnation equilibrium time of the N-TIRs when compared to that of the NIRs ( $57.73 \%$ versus $36.95 \%$ and 5 min versus $240 \mathrm{~min}$ ). It is confirmed that TBP can interact with N235 during the impregnation process, which shorten the adsorption equilibrium time and increases the adsorption capacity of the N-TIRs for $\mathrm{V}(\mathrm{V})$ when compared to that of the NIRs $(6 \mathrm{~h}$ versus $10 \mathrm{~h}$ and $50.95 \mathrm{mg} \cdot \mathrm{g}^{-1}$ versus $46.73 \mathrm{mg} \cdot \mathrm{g}^{-1}$ ). The kinetics fitting results demonstrate that the adsorption of $\mathrm{V}(\mathrm{V})$ onto N-TIRs and NIRs all conform to pseudo-second order kinetic model and chemical reaction is the rate-limiting step of the whole adsorption process. In the meanwhile, the reaction constant $\left(K_{\mathrm{s}}\right)$ implies that the chemical reaction rate of $\mathrm{V}(\mathrm{V})$ with the impregnated extractants in N-TIRs is faster than that in NIRs. The N-TIRs present higher stability and selectivity than NIRs. This study manifests that the addition of a secondary reagent may be a potential and novel technique on the preparation of SIRs and the enhancement of adsorption and separation for ions.
\end{abstract}

Keywords: solvent-impregnated resins; trialkylamine; tributyl phosphate; vanadium; separation; kinetics

\section{Introduction}

Solvent-impregnated resins (SIRs) that were prepared by incorporating extractants onto polymeric matrix have been widely applied in the fields of separation and purification for metals [1-3] and organics [4,5] from aqueous solutions, due to their high selectivity, operational simplicity, and environmental friendliness [6,7], which can be traced back to the pioneering work that was conducted by Warshawsky [8], Grinstead [9], and Kroebel and Meyer [10] in the early 1970s. Thus, the preparation and application of the SIRs in analytical and environmental chemistry, hydrometallurgy, and radiochemistry fields are still intensive research topics [11]. However, there are some problems that are embedded in SIRs, such as time-consuming of the impregnation process for the extractants impregnating onto the macroporous matrix, long time of the adsorption equilibrium and low 
adsorption capacity of the SIRs for metal ions, which may impede the industrial application of SIRs. For instance, Liang et al. [6] investigated the adsorption and separation of V(IV) from the vanadium leaching solution containing Fe(III) and $\mathrm{Al}(\mathrm{III})$ with D2EHPA-impregnated resins (DIRs). It was found that the impregnation equilibrium time exceeds $24 \mathrm{~h}$ and the adsorption capacity of the DIRs for vanadium (IV) is about $16.0 \mathrm{mg} \cdot \mathrm{g}^{-1}$ after a $18 \mathrm{~h}$ long reaction. Navarro et al. [12] prepared the SIRs containing Cyanex 921 and used it to adsorb Cd. The results showed that the impregnation process is time-consuming (about $24 \mathrm{~h}$ ) and the SIRs just have an adsorption capacity of $13 \mathrm{mg} \cdot \mathrm{g}^{-1}$ for Cd after $18 \mathrm{~h}$ adsorption.

Tributyl phosphate (TBP), as a common extractant and modifier, is widely applied in the solvent extraction of metals. Some researchers have proved that the addition of TBP to D2EHPA can improve the phase separation and extraction efficiency [13-15]. For example, Ma et al. [13] studied the separation of V(IV) and Fe(III) from the acid leach solution of stone coal by D2EHPA/TBP, and found that V(IV) and Fe(III) can be effectively separated while using the combination of D2EHPA and TBP. In addition, fewer stages could be used to achieve the required zinc recovery and its effective separation from solutions containing other metal ions by using TBP as a modifier [16]. Based on the fact that a positive synergic effect on the extraction of targeted metals can be achieved by introducing a secondary reagent, such as TBP, Zheng et al. [17] introduced TBP into the DIRs and investigated the adsorption performance of the D2EHPA-TBP impregnated resins (D-TIRs) for V(IV). The results revealed that the D-TIRs have higher adsorption capacity for $\mathrm{V}(\mathrm{IV})$ at shorter adsorption equilibrium time and have better separation capability of V(IV) from Fe(II) and Al(III) when compared with DIRs.

In our previous work, we have prepared trialkylamine N235-impregnated resins (NIRs) and used it for the adsorption of vanadium on account of their high affinity to $\mathrm{V}(\mathrm{V})$ in a low $\mathrm{pH}$ environment [18]. As is known to us, there are few published papers in open literature on the addition of TBP into NIRs and the adsorption characteristics of such prepared SIRs for V(V). In this study, the objective of introducing TBP into NIRs is to improve the performance of the extractant and resolve the aforementioned problems concerning to SIRs. The effects of molar ratio of N235 to TBP, extractants concentration and impregnation time on the impregnation ratio and adsorption performance of the N235-TBP impregnated resins (N-TIRs) for V(V) were studied. The adsorption isotherms and kinetics of $\mathrm{V}(\mathrm{V})$ were investigated, so as to analyze the improvement mechanism for $\mathrm{V}(\mathrm{V})$ adsorption onto N-TIRs. In addition, the selectivity and stability of the N-TIRs were also investigated in the adsorption process. The study may propose a new sight on the preparation of SIRs by introducing a secondary reagent and promote the development and application of SIRs in the fields of separation and recovery of metals from aqueous solution.

\section{Materials and Methods}

\subsection{Materials}

Trialkylamine (N235, $\mathrm{R}_{3} \mathrm{~N}, \mathrm{R}=\mathrm{C}_{8}-\mathrm{C}_{10}$ ), as supplied by Qinshi Technology Co., Ltd., Zhengzhou, China, was used as the primary extractant. Tributyl phosphate (TBP) was purchased from Sinopharm Chemical Reagent Co., Ltd., Shanghai, China. The petroleum ether with a boiling point range of $60-90{ }^{\circ} \mathrm{C}$ (Sinopharm Chemical Reagent Co., Ltd., Shanghai, China) was used to dilute the mixture of $\mathrm{N} 235$ and TBP for preparing SIRs. The pure V(V) solution was prepared by dissolving $\mathrm{V}_{2} \mathrm{O}_{5}$ in the diluted $\mathrm{H}_{2} \mathrm{SO}_{4}$ solutions $(\mathrm{pH}=0.8)$ to keep the concentration of $\mathrm{V}(\mathrm{V})$ at about $1350 \mathrm{mg} \cdot \mathrm{L}^{-1}$. The simulated vanadium-bearing solutions containing $1350 \mathrm{mg} \cdot \mathrm{L}^{-1}$ vanadium, $9450 \mathrm{mg} \cdot \mathrm{L}^{-1}$ aluminum, $2025 \mathrm{mg} \cdot \mathrm{L}^{-1}$ iron, $405 \mathrm{mg} \cdot \mathrm{L}^{-1}$ phosphorus, and $135 \mathrm{mg} \cdot \mathrm{L}^{-1}$ silicon, which refers to the actual acid leaching solution [19], were prepared by two steps. Firstly, the $\mathrm{pH}$ of the pure $\mathrm{V}(\mathrm{V})$ solution was adjusted to about 1.6 while using concentrated sodium hydroxide. Secondly, some amounts of $\mathrm{Al}_{2}\left(\mathrm{SO}_{4}\right)_{3} \cdot 18 \mathrm{H}_{2} \mathrm{O}, \mathrm{Fe}_{2}\left(\mathrm{SO}_{4}\right)_{3} \cdot 9 \mathrm{H}_{2} \mathrm{O}, \mathrm{Na}_{3} \mathrm{PO}_{4} \cdot 12 \mathrm{H}_{2} \mathrm{O}$ and $\mathrm{Na}_{2} \mathrm{SiO}_{3} \cdot 9 \mathrm{H}_{2} \mathrm{O}$ (purchased from Sinopharm Chemical Reagent Co., Ltd., Shanghai, China) were added into the pure V(V) solution, subsequently, the mixtures were filtrated after that the solutions were clear. Then, the $\mathrm{pH}$ of the 
filtrate was adjusted to 1.8 by using concentrated sulfuric acid. All of these chemical reagents are of analytical grade.

Macroporous resin Amberlite ${ }^{\circledR}$ XAD-16HP provided by Shanghai Anland Co., Ltd. (Shanghai, China), was used as a support resin for preparing SIRs. The physical characteristics of XAD-16HP resins are shown in Table 1. The resins were screened to keep the size range of $0.425-0.850 \mathrm{~mm}$ and were pretreated, as follows, for the preparation of SIRs [20]. Firstly, the resins were soaked in ethanol for $4 \mathrm{~h}$ to remove the remaining monomers and other types of impurities, followed by washing with deionized water until the effluent was clear, and then filtered and dried at $60^{\circ} \mathrm{C}$ in a vacuum oven for $12 \mathrm{~h}$.

Table 1. Physical characteristics of XAD-16HP resin.

\begin{tabular}{cc}
\hline Parameters & Value \\
\hline Pore volume & $1.82 \mathrm{~mL} \cdot \mathrm{g}^{-1}$ \\
Specific surface area & $\geq 800 \mathrm{~m}^{2} \cdot \mathrm{g}^{-1}$ \\
Average pore diameter & $15 \mathrm{~nm}$ \\
Particle size & $0.425-0.850 \mathrm{~mm}$ \\
Matrix & Phenyl ethylene-divinyl benzene \\
\hline
\end{tabular}

\subsection{Preparation of SIRs}

N235 was firstly mixed with TBP according to different molar ratios and the mixtures were diluted by petroleum ether, then the diluted N235-TBP were mixed with the pretreated resins at the liquid to solid ratio of $25: 1 \mathrm{~mL} \cdot \mathrm{g}^{-1}$. Subsequently, the mixtures were shaken at $25^{\circ} \mathrm{C}$ in a constant temperature bath oscillator (SHA-2, Jintan Yitong Electronic Co., Ltd., Jintan, China) for a different amount of time. After the impregnation, the resins were separated using a Buchner funnel and then washed with deionized water until the effluent was clear. Finally, the obtained N-TIRs were heated at $60^{\circ} \mathrm{C}$ in a vacuum oven for $12 \mathrm{~h}$ to remove the remaining diluent prior to use. The impregnation ratio of the N-TIRs, $\eta(\%)$, i.e. the ratio of the loaded extractants onto the SIRs, was calculated according to Equation (1):

$$
\eta=\frac{m_{2}-m_{1}}{m_{2}} \times 100 \%
$$

where $m_{1}$ is the weight of the pretreated resins $(\mathrm{g}), m_{2}$ is the weight of the dry N-TIRs $(\mathrm{g})$.

\subsection{Static Adsorption Experiments}

All of the static adsorption experiments were conducted, unless specified, by using the pure $V(V)$ solution. First, the N-TIRs were added to the $\mathrm{V}(\mathrm{V})$-bearing solution (as the liquid to solid ratio of $\left.20: 1 \mathrm{~mL} \cdot \mathrm{g}^{-1}, \mathrm{pH}=1.8\right)$ in a conical flask, then the mixture was shaken (120 rpm) in a bath oscillator at $25{ }^{\circ} \mathrm{C}$ for different contacting time. Finally, the resins were filtered and the adsorption capacity of the SIRs for $\mathrm{V}(\mathrm{V})\left(Q_{\mathrm{M}}\right)$, the distribution coefficient $(D)$, and separation factor $(\beta)$, were calculated according to Equations (2)-(4), respectively:

$$
\begin{gathered}
Q_{\mathrm{M}}=\frac{\left(C_{0}-C_{\mathrm{e}}\right) V}{m_{2}} \\
D=\frac{Q_{\mathrm{e}}}{C_{\mathrm{e}}} \\
\beta_{\mathrm{A} / \mathrm{B}}=\frac{D_{\mathrm{A}}}{D_{\mathrm{B}}}
\end{gathered}
$$

where $V$ is the volume of $\mathrm{V}(\mathrm{V})$ solution $(\mathrm{L}), C_{0}$ and $C_{\mathrm{e}}$ are initial and equilibrium concentrations of $\mathrm{V}(\mathrm{V})$ in the solutions (mg. $\left.\mathrm{L}^{-1}\right)$, respectively, and $m_{2}$ is the weight of the dry N-TIRs $(\mathrm{g}) . Q_{\mathrm{e}}$ is the equilibrium adsorption capacity of the N-TIRs for metal ions $\left(\mathrm{mg} \cdot \mathrm{g}^{-1}\right) . D_{\mathrm{A}}$ and $D_{\mathrm{B}}$ are the distribution 
coefficient of $\mathrm{V}(\mathrm{V})$ and impurity ion between $\mathrm{N}$-TIRs and the aqueous solution, respectively. $\beta_{\mathrm{A} / \mathrm{B}}$ represents the separation factor of ion A over B.

\subsection{Cyclic Adsorption Experiments}

The N-TIRs were separated from the $\mathrm{V}(\mathrm{V})$-bearing solutions after adsorption equilibrium, and they were then added to $40 \mathrm{~mL} \mathrm{Na}_{2} \mathrm{CO}_{3}$ solutions with a mass concentration of $18 \mathrm{wt} \%$. After shaking for $12 \mathrm{~h}$ at room temperature, the N-TIRs were filtered and washed with dilute sulfuric acid $(\mathrm{pH}=1.8)$ until the $\mathrm{pH}$ value of the eluent was about 1.8 and were re-used for the next static adsorption for $\mathrm{V}(\mathrm{V})$, the process of which was the same as that depicted in Section 2.3.

\subsection{Analytical and Testing Methods}

The concentration of $\mathrm{V}(\mathrm{V})$ in the solution was determined by ferrous ammonium sulfate titration while using 2-(phenylamino)-benzoic acid as indicator [21]. The concentration of other metal ions in the aqueous solutions was determined by Inductively Coupled Plasma Atomic Emission Spectrometer (Optima4300DV, PerkinElmer, Waltham, MA, USA). The change of characteristic absorption peaks of different SIRs was obtained by Fourier transform infrared spectroscopy (VERTEX 70, Bruker, Germany). The N-TIRs and NIRs after adsorption of $\mathrm{V}(\mathrm{V})$ were carefully split by blade from the core and the cross section of resins were inspected and scanned by scanning electron microscopy (SEM, JSM-IT300, JEOL Co., Tokyo, Japan), equipped with an energy dispersive spectrometer (EDS, Oxford, UK) to observe the vanadium distribution in the pores of the SIRs.

\section{Results and Discussion}

\subsection{Effects of Impregnation Conditions on the N-TIRs}

\subsubsection{Effects of the Used Mixtures}

The effects of the molar ratio of N235 to TBP on the impregnation ratio of N-TIRs and the adsorption capacity for $\mathrm{V}(\mathrm{V})$ are shown in Figure 1.

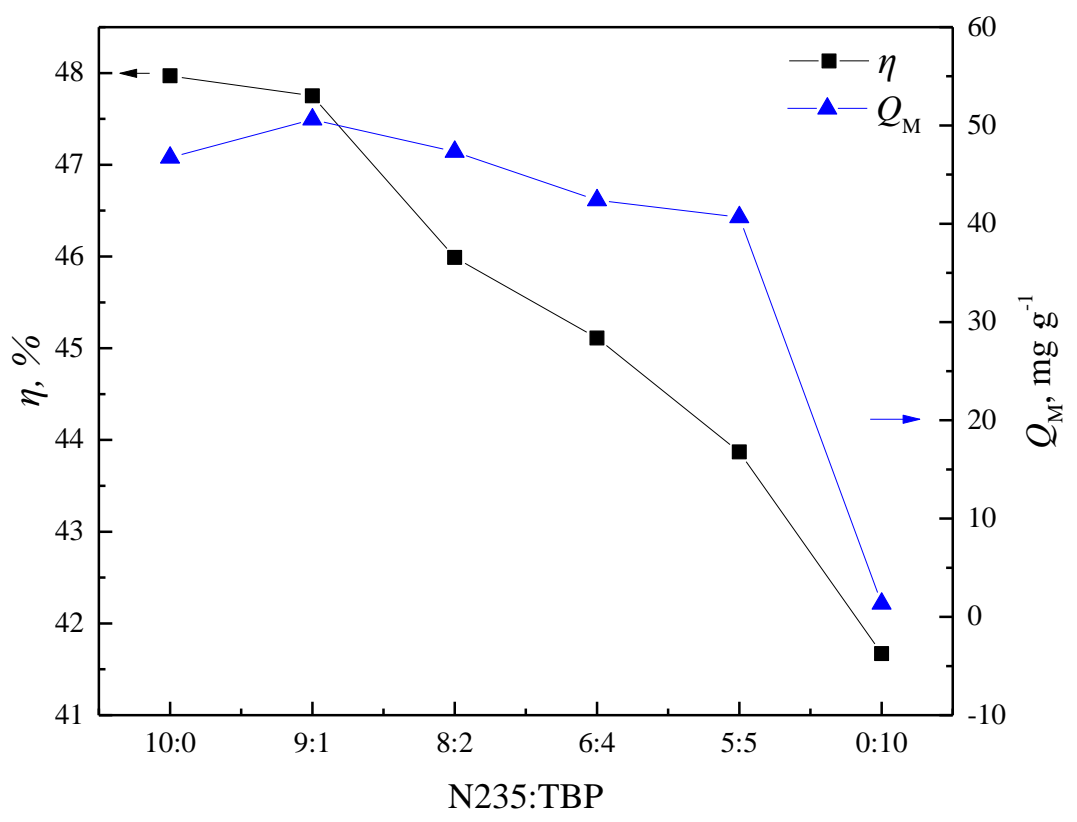

Figure 1. Effects of the molar ratio of N235 to tributyl phosphate (TBP) on the preparation of N235-TBP impregnated resins (N-TIRs) and adsorption capacity for $\mathrm{V}(\mathrm{V})$ (Extractants concentration $=1.0 \mathrm{~mol} \cdot \mathrm{L}^{-1}$ ) $\left(C_{0}(\mathrm{~V})=1200 \mathrm{mg} \cdot \mathrm{L}^{-1}, 14 \mathrm{~h}\right.$ adsorption time, $\left.25^{\circ} \mathrm{C}\right)$. 
Figure 1 shows that the impregnation ratio of the N-TIRs significantly decline with the decrease of the molar ratio of N235 to TBP, which may be attributed to the different affinity of N235 and TBP for the support resins. As is known to us, the weak polar N235 have relatively higher affinity for the non-polar matrix of the resins matrix (Phenyl ethylene-divinyl benzene) in comparison to polar TBP [17]. As the molar ratio of N235 to TBP decreases, the content of N235 decreases and thus the impregnation ratio of the N-TIRs decline with the decreasing molar ratio. The adsorption capacity of the N-TIRs for $\mathrm{V}(\mathrm{V})$ firstly increases and then decreases with the decrease of the molar ratio. As shown in Figure 1, the decrease of the impregnation ratio is not obvious when the molar ratio of N235 to TBP declines from 10:0 to 9:1, but the adsorption capacity increases somewhat, which may be ascribed to that the addition of TBP improves the extractability of N235 for V(V). However, the amount of effective N235 declines when the molar ratio of N235 to TBP decreases from 9:1 to 0:10, thus the adsorption capacity of the N-TIRs for $\mathrm{V}(\mathrm{V})$ declines. As a result, the optimal molar ratio of N235 to TBP was selected as 9:1 for the following adsorption experiments.

\subsubsection{Effects of Extractants Concentration}

Figure 2 shows the change of the impregnation ratio and adsorption capacity for $V(V)$, along with the extractants concentration.

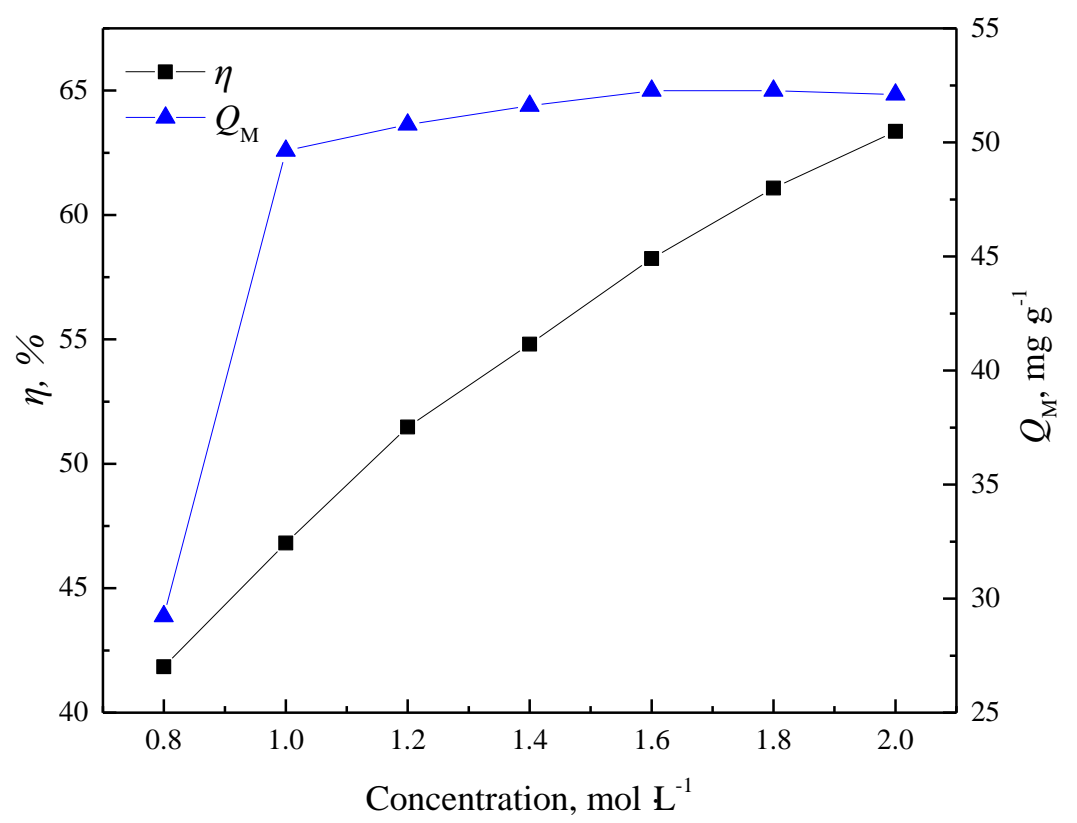

Figure 2. Effects of extractants concentration on the impregnation ratio of N-TIRs and adsorption capacity for $\mathrm{V}(\mathrm{V})(\mathrm{N} 235$ : $\mathrm{TBP}=9: 1)$.

The results show that the impregnation ratio $(\eta)$ increase approximately linearly with the increase of the extractants concentration, while the adsorption capacity of the N-TIRs for V(V) firstly increases sharply and then tends to reach saturation with the increasing extractants concentration. According to our previous work [7], the extractant is preferentially adsorbed in the micropores as wall-spreading to form a monolayer, resulting in the relatively lower impregnation ratio at low extractants concentration. Whereas, XAD-16HP resin that was used in this study is mainly composed of mesopores and macropores, with the increasing content of the extractants, the extractants more likely accumulate in these pores as pore-filling to form multilayer, which lead to the higher impregnation ratio at high extractants concentration. It is interesting to observe that the adsorption capacity of the N-TIRs for $V(V)$ just increase slowly though the impregnation ratio of the N-TIRs obviously increase as the extractants increase from 1.0 to $2.0 \mathrm{~mol} \cdot \mathrm{L}^{-1}$. As more extractants accumulate in the mesopores and macropores as 
multilayer, the impregnation ratio of the N-TIRs is close to the peak (59.9\%), which results in that the effective $\mathrm{N} 235$ reacting with $\mathrm{V}(\mathrm{V})$ does not increase remarkably in spite of the augment of the loaded extractant [20]. No significance difference is observed in the adsorption capacity when the extractants concentration is higher than $1.6 \mathrm{~mol} \cdot \mathrm{L}^{-1}$ (Figure 2). Therefore, the optimal extractants concentration is selected as $1.6 \mathrm{~mol} \cdot \mathrm{L}^{-1}$ in the impregnation process.

\subsubsection{Effects of Impregnation Time}

The impregnation ratio and the adsorption capacity of the N-TIRs at different impregnation times, ranging from $5 \mathrm{~min}$ to $360 \mathrm{~min}$, are shown in Figure 3.

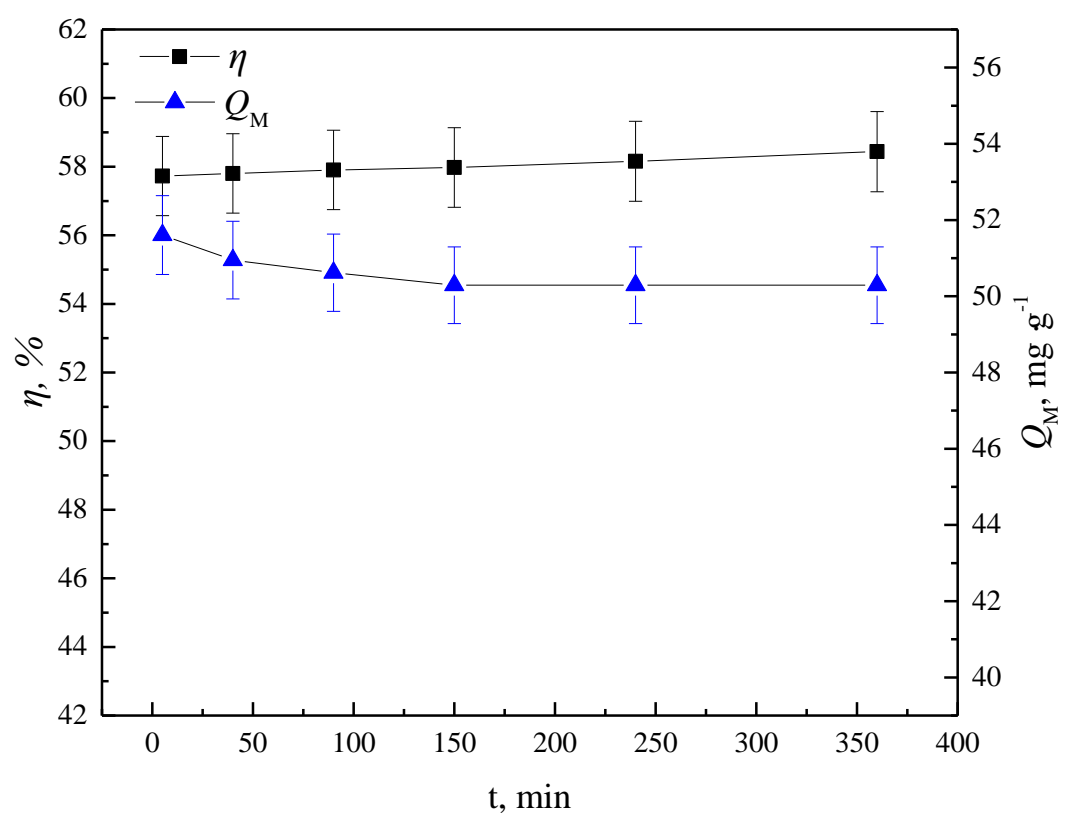

Figure 3. Effects of impregnation time on the impregnation ratio of N-TIRs and adsorption capacity for $\mathrm{V}(\mathrm{V})\left(\mathrm{N} 235: \mathrm{TBP}=9: 1\right.$, Extractants concentration $\left.=1.6 \mathrm{~mol} \cdot \mathrm{L}^{-1}\right)$.

It is observed from Figure 3 that the impregnation ratio just increases slightly (from $57.73 \%$ to $59.12 \%$ ) with the impregnation time increasing from $5 \mathrm{~min}$ to $360 \mathrm{~min}$. The results indicate that the impregnation process of the N-TIRs accomplish faster (about $5 \mathrm{~min}$ ) than that of the NIRs (about $240 \mathrm{~min}$ ) [19], attributing to the addition of TBP [22]. The "interface modifier", TBP, can improve the phase separation rate, i.e., accelerate the molecular motion rate between the aqueous and organic phases [14,23], thus the addition of TBP can obviously shorten the impregnation equilibrium time of the N-TIRs. As is presented in Figure 3, the adsorption capacity of N-TIRs for V(V) firstly slowly declines and then keeps constant with the increasing impregnation ratio. Based on the discussion in Section 3.1.2, the impregnation ratio of the N-TIRs approach to the peak value (59.9\%), resulting in that the extractant effectively reacting with $V(V)$ just slightly decrease, thus the adsorption capacity of N-TIRs for $\mathrm{V}(\mathrm{V})$ firstly slowly declines and then keeps constant.

\subsection{Characterizations of N-TIRs and NIRs}

\subsubsection{FTIR Analyses}

Figure 4 presents the FTIR spectra of the N-TIRs and NIRs. A literature shows that the characteristic vibration bands of $\mathrm{P}=\mathrm{O}, 1281 \mathrm{~cm}^{-1}$, would appear in the SIRs containing TBP [17]. It is obviously found that the characteristic adsorption peak of $\mathrm{N} 235\left(\mathrm{C}-\mathrm{N}\right.$ bonds, $\left.1099 \mathrm{~cm}^{-1}\right)$ shifts to the lower wavenumber $\left(1096 \mathrm{~cm}^{-1}\right)$ and the intensity of the $\mathrm{C}-\mathrm{N}$ bonds decreases (Figure $4 \mathrm{a}$ ), 
and the $\mathrm{P}=\mathrm{O}$ stretching bands also shifts to lower wavenumber $1263 \mathrm{~cm}^{-1}$ (Figure $4 \mathrm{~b}$ ), which may be attributed to that N235 is involved with TBP [23]. Therefore, it may be concluded that an interaction between TBP and N235 has occurred during the impregnation process, rendering the improvement of the impregnation rate and the impregnation ratio of the extractants onto the resins.

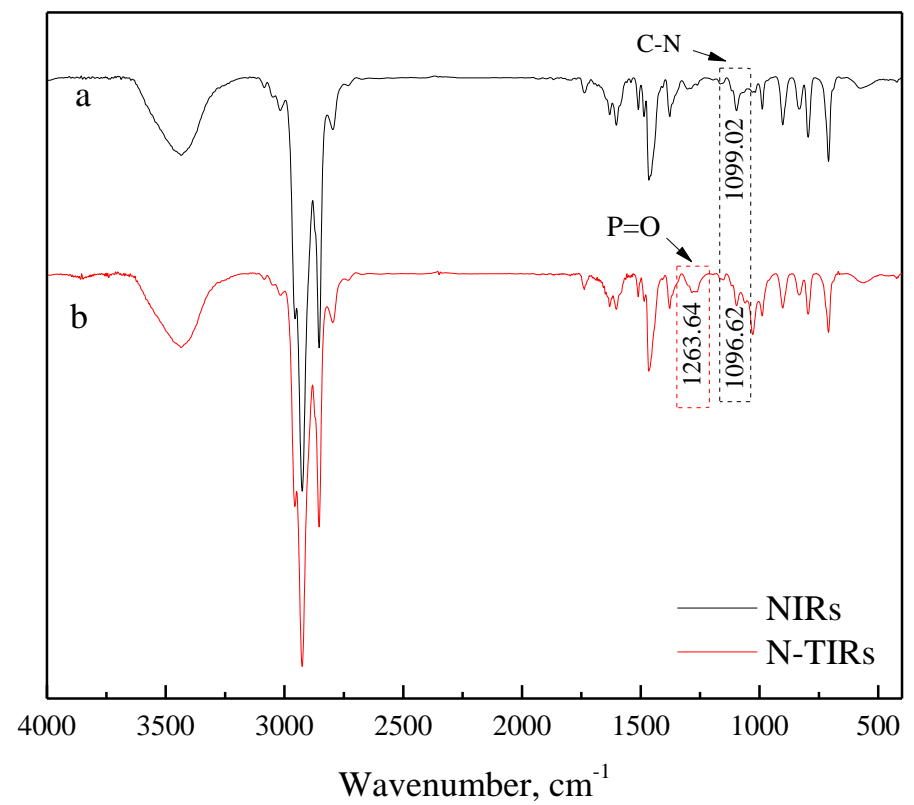

Figure 4. FTIR spectra of N235-impregnated resins (NIRs) (a) and N-TIRs (b).

\subsubsection{SEM-EDS Analyses}

Figure 5 presents the distribution of $\mathrm{V}(\mathrm{V})$ on the cross section of NIRs and N-TIRs after the adsorption of $\mathrm{V}(\mathrm{V})$. It can be obviously seen from the cross section of NIRs (Figure 5(a2)) and N-TIRs (Figure 5(b2)) that V(V) distribute deeper into the pores of the N-TIRs than in NIRs, implying that the more extractants reacting with $\mathrm{V}(\mathrm{V})$ can enter deeper into the pores of the resins during the impregnation process by adding TBP, which is consistent with the fact that the impregnation ratio of N-TIRs is higher than that of NIRs. In the meanwhile, the distribution of $V(V)$ on the cross section also verifies that more $\mathrm{V}(\mathrm{V})$ are loaded onto the N-TIRs than onto NIRs.
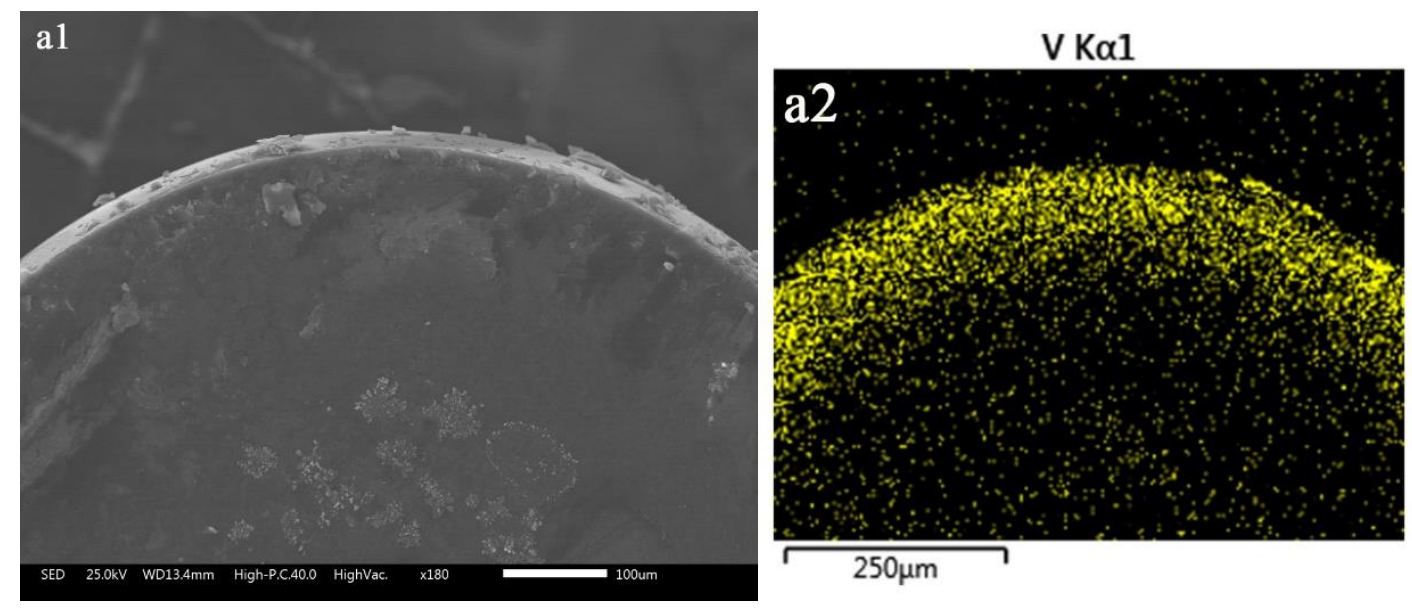

Figure 5. Cont. 

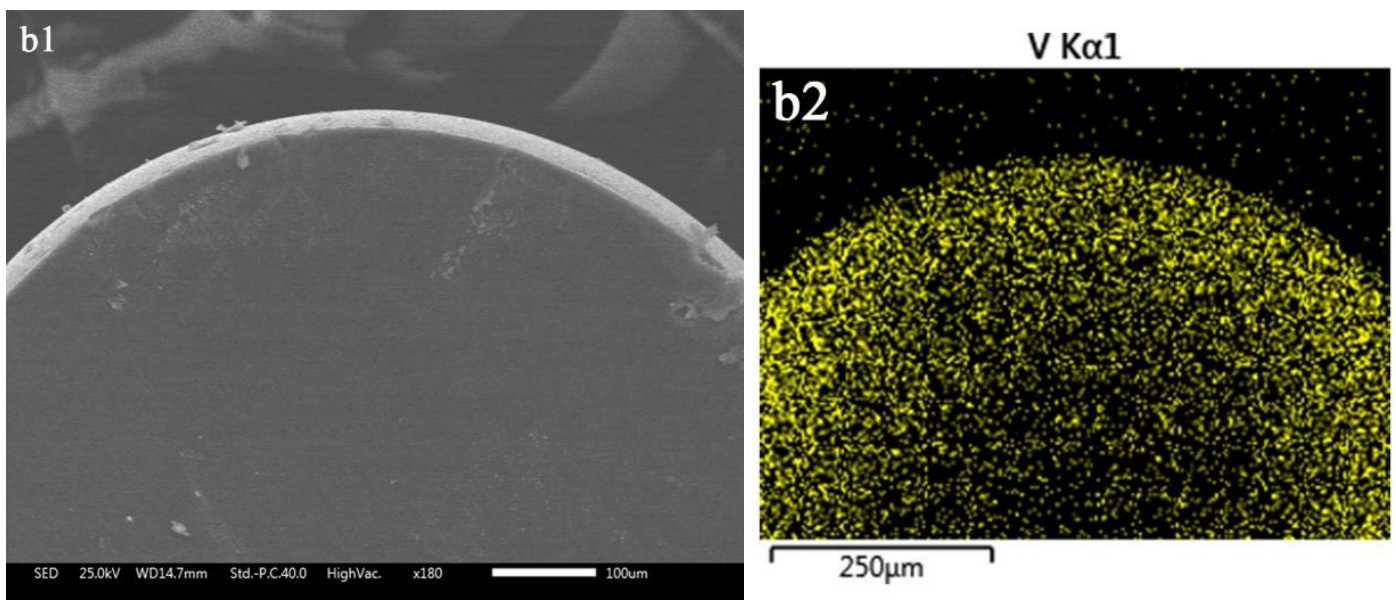

Figure 5. Scanning electron microscopy-energy dispersive spectrometer (SEM-EDS) plane scan analyses of the cross section of NIRs (a1): the morphologies; (a2): the distribution of V(V) and N-TIRs (b1): the morphologies; (b2): the distribution of $\mathrm{V}(\mathrm{V})$ after adsorption of $\mathrm{V}(\mathrm{V})$.

\subsection{Adsorption of $V(V)$ onto the N-TIRs and NIRs}

\subsubsection{Effects of Initial V(V) Concentration}

The adsorption of $\mathrm{V}(\mathrm{V})$ by N-TIRs and NIRs were conducted at different initial $\mathrm{V}(\mathrm{V})$ concentration, ranging from $100 \mathrm{mg} \cdot \mathrm{L}^{-1}$ to $1350 \mathrm{mg} \cdot \mathrm{L}^{-1}$.

As is found in Figure 6, the adsorption capacity of N-TIRs and NIRs for V(V) both approximately logarithmically grow with the increase of the initial $\mathrm{V}(\mathrm{V})$ concentration. The equilibrium adsorption capacity of the N-TIRs is evidently higher than that of the NIRs when the initial $\mathrm{V}(\mathrm{V})$ concentration exceeds $500 \mathrm{mg} \cdot \mathrm{L}^{-1}$. The higher impregnation ratio of the N-TIRs leads to higher adsorption capacity for $\mathrm{V}(\mathrm{V})$ onto such SIRs than that onto NIRs.

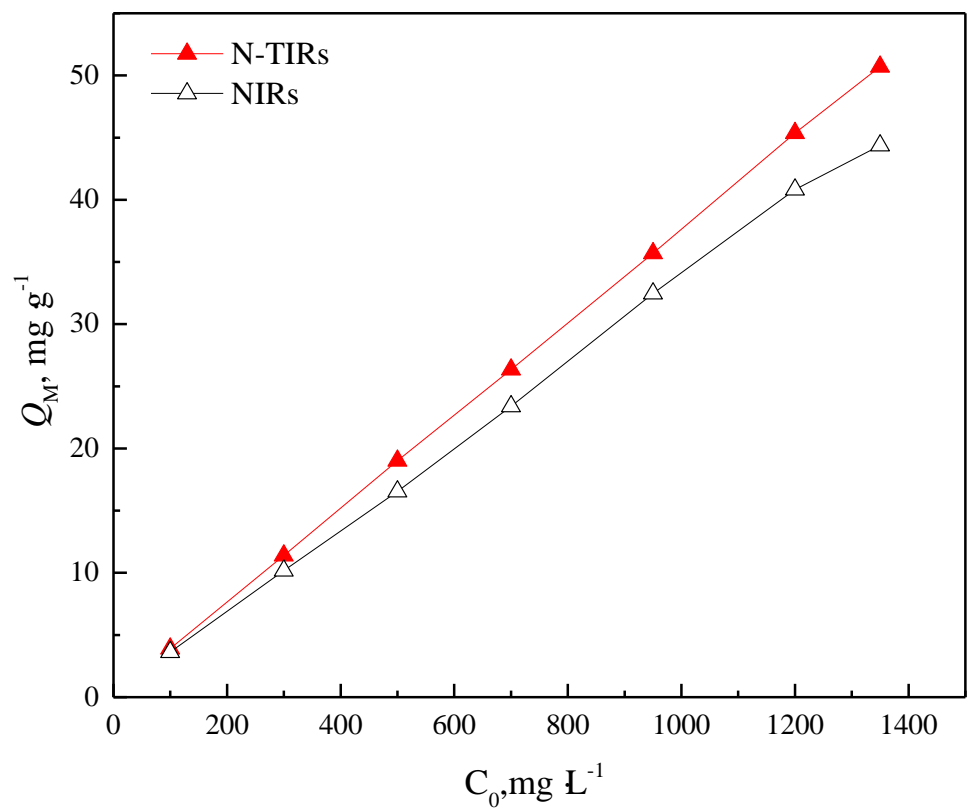

Figure 6. Effects of initial $\mathrm{V}(\mathrm{V})$ concentration on the adsorption capacity of the N-TIRs and NIRs for $V(V)$. 


\subsubsection{Adsorption Isotherm}

In general, the Langmuir and Freundlich isotherm models are widely used to depict the adsorption of metal ions onto SIRs [11,24,25]. Langmuir model is commonly used to describe the uptake of metal ions that occurs on a homogeneous surface by monolayer adsorption without any interaction between the adsorbed ions, and the Freundlich model assumes that the adsorption of metal ions occurs on a heterogeneous surface by monolayer adsorption. The isotherm models are listed as Equations (5) and (6), respectively.

$$
\begin{gathered}
\frac{C_{\mathrm{e}}}{Q_{\mathrm{e}}}=\frac{1}{Q_{0} K_{\mathrm{L}}}+\frac{C_{\mathrm{e}}}{Q_{0}} \\
\log Q_{\mathrm{e}}=\log K_{\mathrm{F}}+\frac{1}{n} \log C_{\mathrm{e}}
\end{gathered}
$$

where $Q_{\mathrm{e}}$ is the amount of adsorbed metal ions per unit mass of SIRs at equilibrium $\left(\mathrm{mg} \cdot \mathrm{g}^{-1}\right), C_{\mathrm{e}}$ is the concentration of metal ions at equilibrium in raffinate $\left(\mathrm{mg} \cdot \mathrm{L}^{-1}\right), Q_{0}$ is the adsorbent capacity (possible maximum amount of metal ions adsorbed per unit mass of SIRs, $\left.\mathrm{mg} \cdot \mathrm{g}^{-1}\right), K_{\mathrm{L}}$ is Langmuir isothermal adsorption equilibrium constant $\left(\mathrm{L} \cdot \mathrm{mg}^{-1}\right)$, and $K_{\mathrm{F}}$ and $n$ are the Freundlich constants for adsorption capacity and adsorption intensity, respectively.

The fitting results of Langmuir and Freundlich isotherm model for N-TIRs and NIRs are shown in Figure 7, and the parameters of Langmuir and Freundlich isotherm models are listed in Table 2.
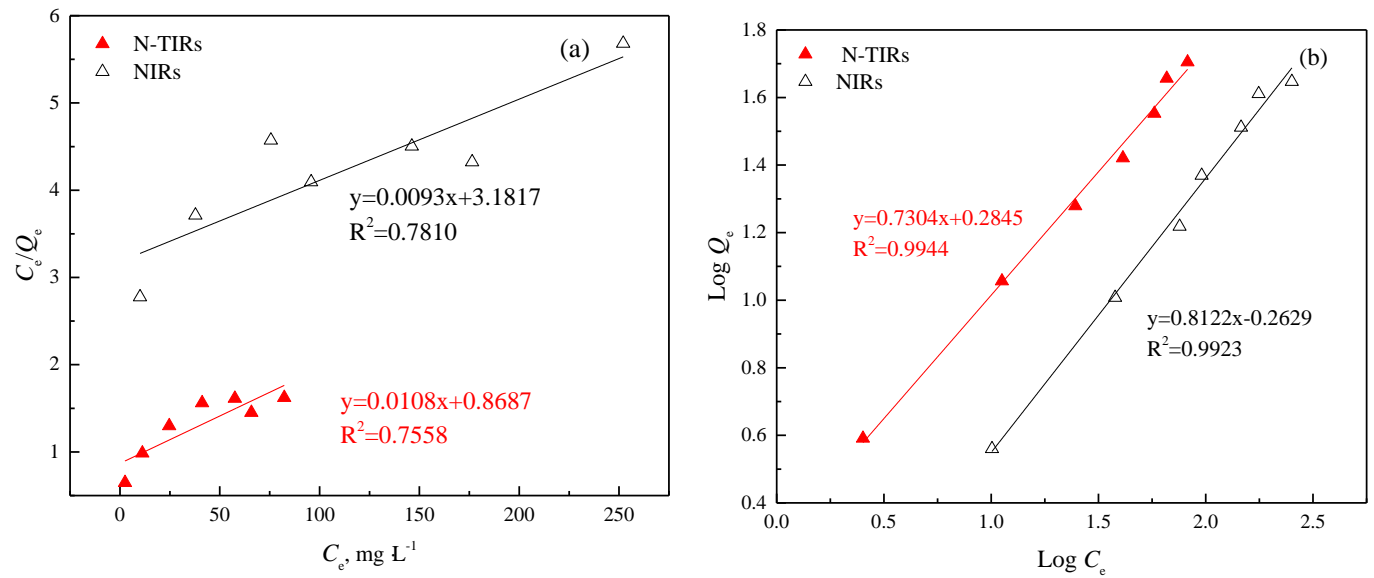

Figure 7. Langmuir (a) and Freundlich (b) isotherm for V(V) adsorption onto N-TIRs and NIRs.

Table 2. Parameters of the Langmuir and Freundlich isotherm models for $V(V)$ adsorption.

\begin{tabular}{ccccccc}
\hline \multirow{2}{*}{ SIRs } & \multicolumn{3}{c}{ Langmuir } & \multicolumn{3}{c}{ Freundlich } \\
\cline { 2 - 7 } & $\boldsymbol{Q}_{\mathbf{0}}$ & $\boldsymbol{K}_{\mathbf{L}}$ & $\boldsymbol{R}^{\mathbf{2}}$ & $\boldsymbol{n}$ & $\boldsymbol{K}_{\mathbf{F}}$ & $\boldsymbol{R}^{\mathbf{2}}$ \\
\hline N-TIRs & 92.59 & 0.0124 & 0.7558 & 1.37 & 1.9253 & 0.9944 \\
NIRs & 107.53 & 0.0029 & 0.7810 & 1.23 & 0.5459 & 0.9923 \\
\hline
\end{tabular}

Figure 7 manifests that the Freundlich isotherm models fits better for $V(V)$ adsorption onto N-TIRs and NIRs when compared with Langmuir isotherm models due to that the goodness of fit $\left(R^{2}\right)$ with Freundlich isotherm for N-TIRs (0.994) and NIRs (0.9923) are closer to 1 than that of the Langmuir isotherm models for the two SIRs. As can be seen from Table 2, the fitting parameters $\left(n\right.$ and $\left.K_{\mathrm{F}}\right)$ for N-TIRs are both higher than that for NIRs, implying that $\mathrm{V}(\mathrm{V})$ is more easily absorbed on the N-TIRs and the adsorption capacity of $\mathrm{V}(\mathrm{V})$ is higher than that of NIRs, which is consistent with the experiment data. 


\subsubsection{Effects of Adsorption Time}

The effects of contacting time on the adsorption capacity of the N-TIRs and NIRs for V(V) are shown in Figure 8.

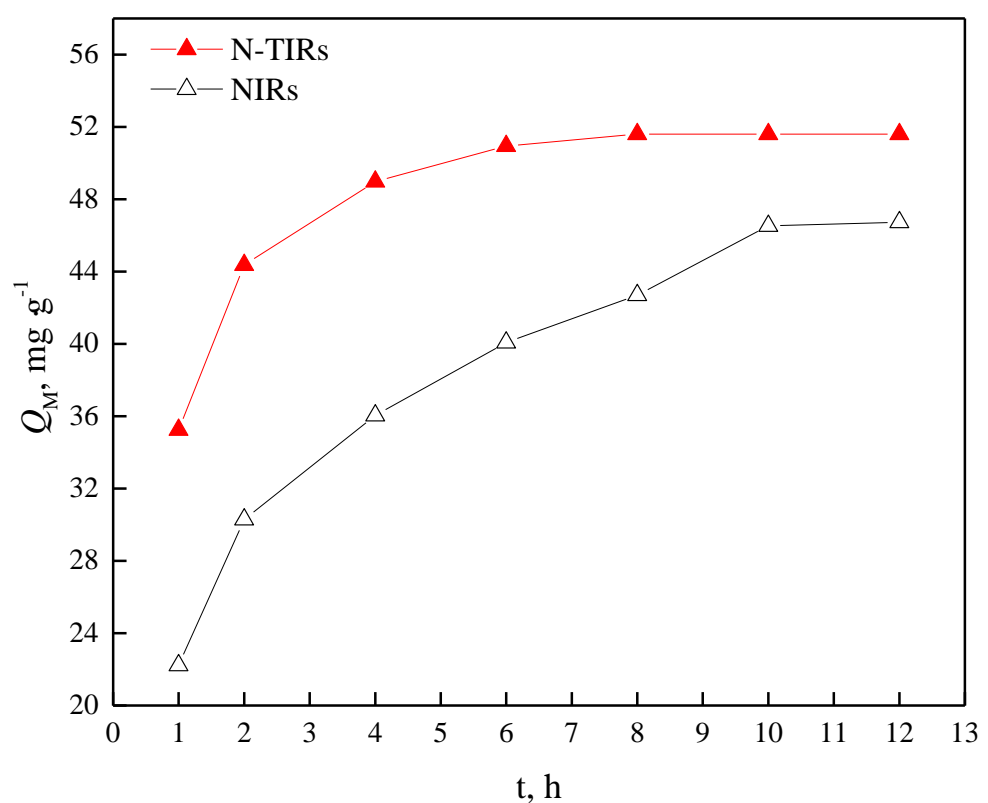

Figure 8. Effects of contacting time on the adsorption capacity of the N-TIRs and NIRs for V(V).

Figure 8 reveals that the adsorption capacity of the N-TIRs and NIRs for V(V) both firstly rapidly increase and then tend to balance with the increasing contacting time. The adsorption capacity of the N-TIRs for $\mathrm{V}(\mathrm{V})$ equilibrates at about $6 \mathrm{~h}$ as $50.95 \mathrm{mg} \cdot \mathrm{g}^{-1}$, but that of the NIRs continuously increases after $6 \mathrm{~h}$, and finally reaches equilibrium at about $10 \mathrm{~h}$ as $46.53 \mathrm{mg} \cdot \mathrm{g}^{-1}$. It is evident that the adsorption capacity of the N-TIRs for $\mathrm{V}(\mathrm{V})$ is always higher than that of the NIRs over the whole contacting time (Figure 8), attributing to the higher impregnation ratio of the N-TIRs in comparison to that of the NIRs, which is also demonstrated in the section "SEM analyses".

\subsubsection{Adsorption Kinetics}

As is mentioned above, the adsorption equilibrium time of N-TIRs is shortened to $6 \mathrm{~h}$ in comparison to that of NIRs (Ca. $10 \mathrm{~h}$ ), implying that the addition of TBP can accelerate the adsorption rate of $\mathrm{V}(\mathrm{V})$ to some extent. The two commonly used kinetic models, pseudo-first order kinetic and pseudo-second order kinetic models [11,24], are used to depict the adsorption process of $\mathrm{V}(\mathrm{V})$ to verify the promotion mechanism for $\mathrm{V}(\mathrm{V})$ adsorption, which are listed as Equations (7) and (8), respectively.

$$
\begin{gathered}
\ln \left(Q_{\mathrm{e}}-Q_{\mathrm{t}}\right)=\ln Q_{\mathrm{e}}-K_{1} t \\
\frac{t}{Q_{\mathrm{t}}}=\frac{1}{K_{2} Q_{e}^{2}}+\frac{t}{Q_{\mathrm{e}}}
\end{gathered}
$$

where $Q_{\mathrm{t}}$ is the adsorption capacity $\left(\mathrm{mg} \cdot \mathrm{g}^{-1}\right)$ for $\mathrm{V}(\mathrm{V})$ onto the SIRs at any moment, $t$ is the adsorption time $(\mathrm{h})$, and $K_{1}\left(\mathrm{~h}^{-1}\right)$ and $K_{2}\left(\mathrm{~mol} \cdot \mathrm{L}^{-1} \cdot \mathrm{h}^{-1}\right)$ are the adsorption rate constant of pseudo-first order kinetic model and pseudo-second kinetic model, respectively.

The fitting results of pseudo-first order kinetic and pseudo-second order kinetic models for the adsorption of $\mathrm{V}(\mathrm{V})$ onto different SIRs using the two kinetic models are shown in Figure 9, and the fitting parameters are listed in Table 3. 

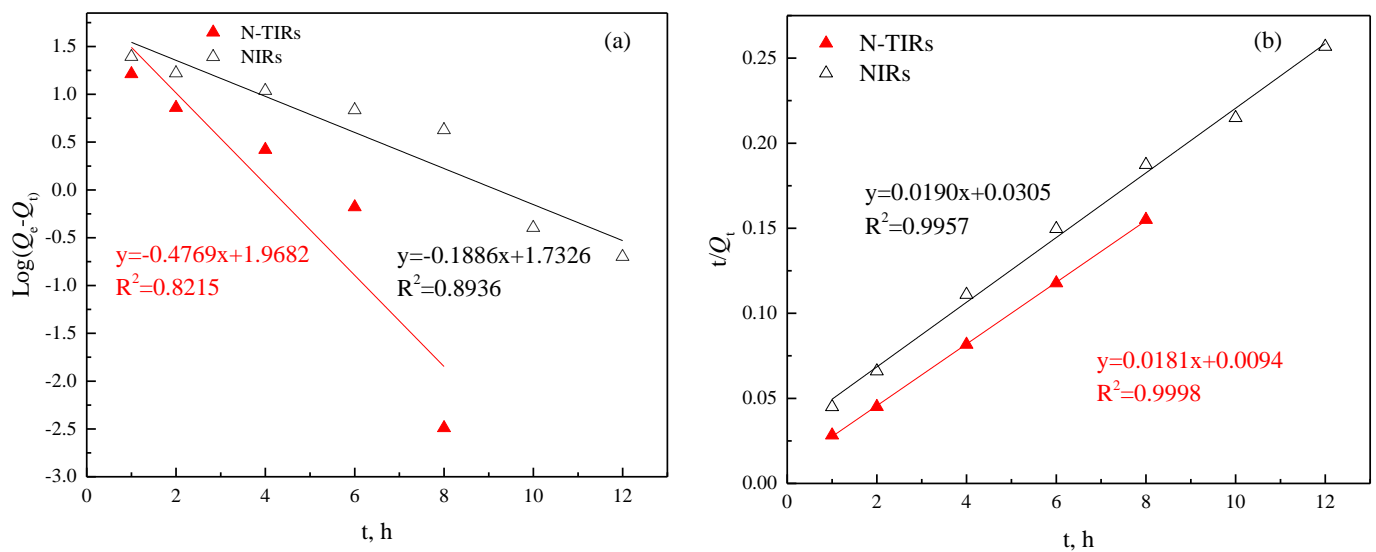

Figure 9. Fitting of the pseudo-first order kinetic (a) and pseudo-second order kinetic model (b) for $\mathrm{V}(\mathrm{V})$ adsorption onto N-TIRs and NIRs.

Table 3. Fitting parameters of the kinetic models for $\mathrm{V}(\mathrm{V})$ adsorption onto the SIRs.

\begin{tabular}{ccccccc}
\hline \multirow{2}{*}{ SIRs } & \multicolumn{3}{c}{ Pseudo-First Order } & \multicolumn{3}{c}{ Pseudo-Second Order } \\
\cline { 2 - 7 } & $\boldsymbol{Q}_{\mathbf{e}}$ & $\boldsymbol{K}_{\mathbf{1}}$ & $\boldsymbol{R}^{\mathbf{2}}$ & $\boldsymbol{Q}_{\mathbf{e}}$ & $\boldsymbol{K}_{\mathbf{2}}$ & $\boldsymbol{R}^{\mathbf{2}}$ \\
\hline N-TIRs & 7.16 & 0.4769 & 0.8251 & 55.25 & 0.0349 & 0.9998 \\
NIRs & 5.66 & 0.1886 & 0.8936 & 52.63 & 0.0118 & 0.9957 \\
\hline
\end{tabular}

The fitting results manifest that the pseudo-second order kinetic model fits better for the adsorption process of $\mathrm{V}(\mathrm{V})$, which indicates that the rate-limiting step of $\mathrm{V}(\mathrm{V})$ adsorption may be chemical sorption or chemisorption involving valency forces through sharing or exchange of electrons between the impregnated extractants and $\mathrm{V}(\mathrm{V})$ [26]. The adsorption rate constant $K_{2}$ of N-TIRs $(0.0349)$ is larger than that of NIRs (0.0118), implying that N-TIRs can reach adsorption equilibrium faster than NIRs, which is identical to the experimental data.

In order to fully confirm the rate limiting step of $\mathrm{V}(\mathrm{V})$ adsorption, the shrinking core model (also called adsorption diffusion models) [11] was used to fit the adsorption processes of $\mathrm{V}(\mathrm{V})$. The relationship between reaction time and the degree of fractional conversion $\left(F=Q_{\mathrm{t}} / Q_{\mathrm{e}}\right)$ are given as Equations (9)-(11) [27]:

(a) When the fluid film diffusion controls:

$$
F=\frac{3 C_{\mathrm{AO}} K_{\mathrm{m} A}}{\alpha r_{0} C_{\mathrm{SO}}} t
$$

(b) when the intraparticle diffusion controls:

$$
3-3(1-F)^{\frac{2}{3}}-2 F=\frac{6 D_{\mathrm{r}} C_{\mathrm{AO}}}{\alpha r_{0}^{2} C_{\mathrm{SO}}} t
$$

(c) when the chemical reaction controls:

$$
1-(1-F)^{\frac{1}{3}}=\frac{K_{\mathrm{S}} C_{\mathrm{AO}}}{r_{0}} t
$$

where $a$ is the stoichiometric coefficient, $C_{\mathrm{SO}}$ is the concentration of solid reactant at the bead's unreacted core $\left(\mathrm{mol} \cdot \mathrm{L}^{-1}\right), C_{\mathrm{AO}}$ is the initial concentration of $\mathrm{V}(\mathrm{V})$ in bulk solution $\left(\mathrm{mol} \cdot \mathrm{L}^{-1}\right), K_{\mathrm{mA}}$ is the mass transfer coefficient of $\mathrm{V}(\mathrm{V})$ through the liquid film $\left(\mathrm{m} \cdot \mathrm{s}^{-1}\right), K_{\mathrm{s}}$ is the reaction constant based on surface $\left(\mathrm{m} \cdot \mathrm{s}^{-1}\right)$, and $r_{0}$ is the radius of SIRs $(\mathrm{m})$. 
Figure 10 shows the fitting results for the adsorption of $\mathrm{V}(\mathrm{V})$ by the shrinking core model, and the fitting parameters are listed in Table 4. It is evident that the chemical reaction kinetic model fits the adsorption of $\mathrm{V}(\mathrm{V})$ better than other models, verifying that the chemical reaction is the rate-limiting step of $\mathrm{V}(\mathrm{V})$ adsorption. In the meantime, the reaction constant $K_{\mathrm{s}}$ of N-TIRs $\left(2.06 \times 10^{-3}\right)$ is larger than that of the NIRs $\left(1.36 \times 10^{-3}\right)$, indicating that the chemical reaction rate of $\mathrm{V}(\mathrm{V})$ with the extractants in the pores of the N-TIRs is faster than that in the pores of the NIRs, which may be ascribed to the fact that the interaction of N235 and TBP improves the extractability of the extractants.
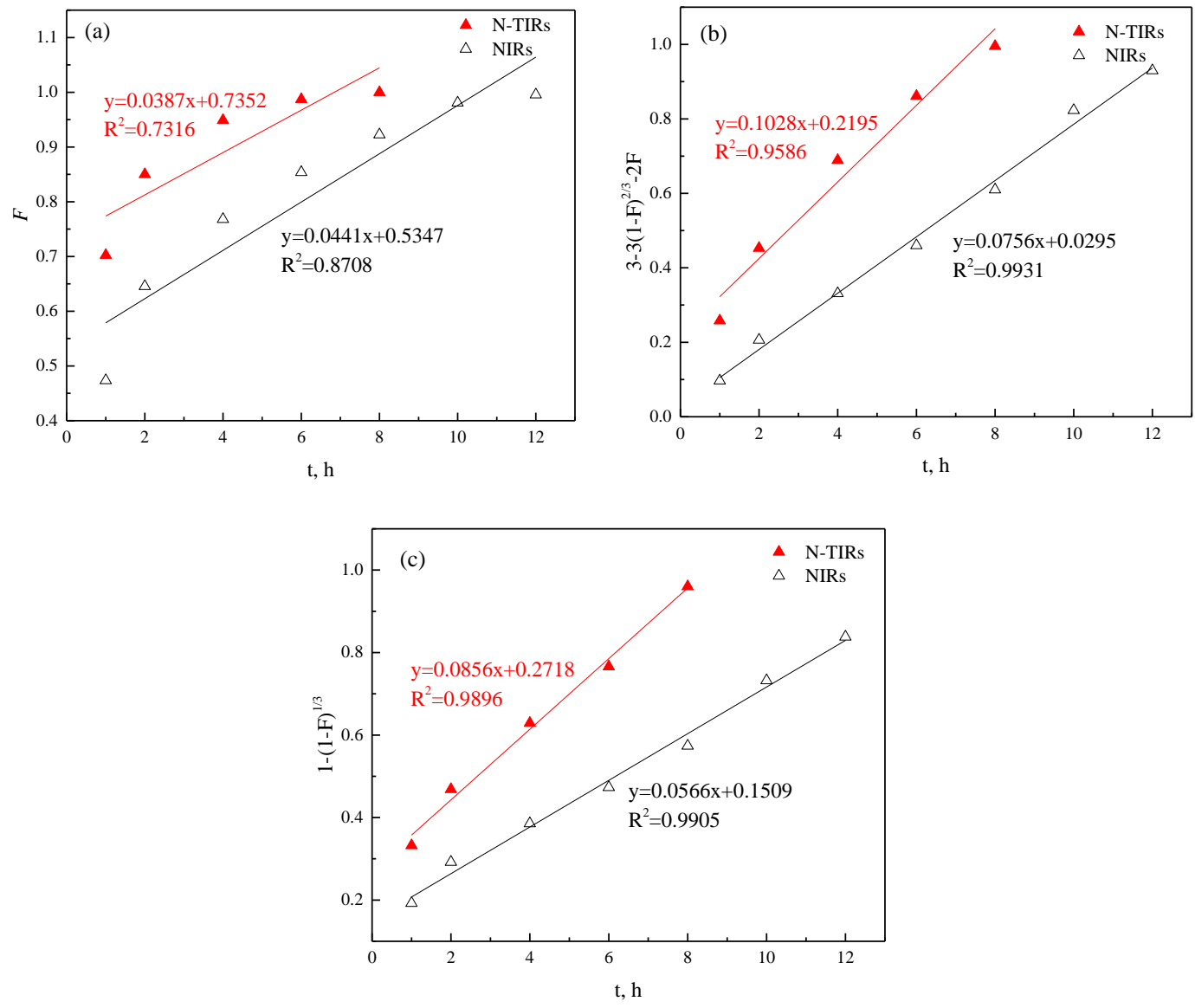

Figure 10. Fitting for the adsorption of $V(V)$ by Shrinking core mode (a) fluid film diffusion, (b) intraparticle diffusion, and (c) chemical reaction.

Table 4. Parameters of the shrinking core model for $V(V)$ adsorption onto the solvent-impregnated resins (SIRs).

\begin{tabular}{ccccc}
\hline \multirow{2}{*}{ SIRs } & \multicolumn{3}{c}{$\boldsymbol{R}^{\mathbf{2}}$} & \multirow{2}{*}{$\boldsymbol{K}_{\mathbf{S}}$} \\
\cline { 2 - 4 } & Fluid Film Diffusion & Intraparticle Diffusion & Chemical Reaction & \\
\hline N-TIRs & 0.7316 & 0.9586 & 0.9896 & $2.06 \times 10^{-3}$ \\
NIRs & 0.8708 & 0.9931 & 0.9905 & $1.36 \times 10^{-3}$ \\
\hline
\end{tabular}

\subsection{Separation of $V(V)$ from Complex Solution}

The separation factor $(\beta)$ for $V(V)$ over impurities onto N-TIRs and NIRs are depicted in Figure 11. It is found that the separation factors for $\mathrm{V}(\mathrm{V})$ over impurities onto N-TIRs are all obviously higher than that of NIRs, indicating that the selectivity of the N-TIRS is superior to that of NIRs, which is attributed to that the addition of TBP can obviously improve the extractability of N235 that is loaded onto the N-TIRs for $\mathrm{V}(\mathrm{V})$. In conclusion, the selectivity of NIRs is improved by adding TBP. 


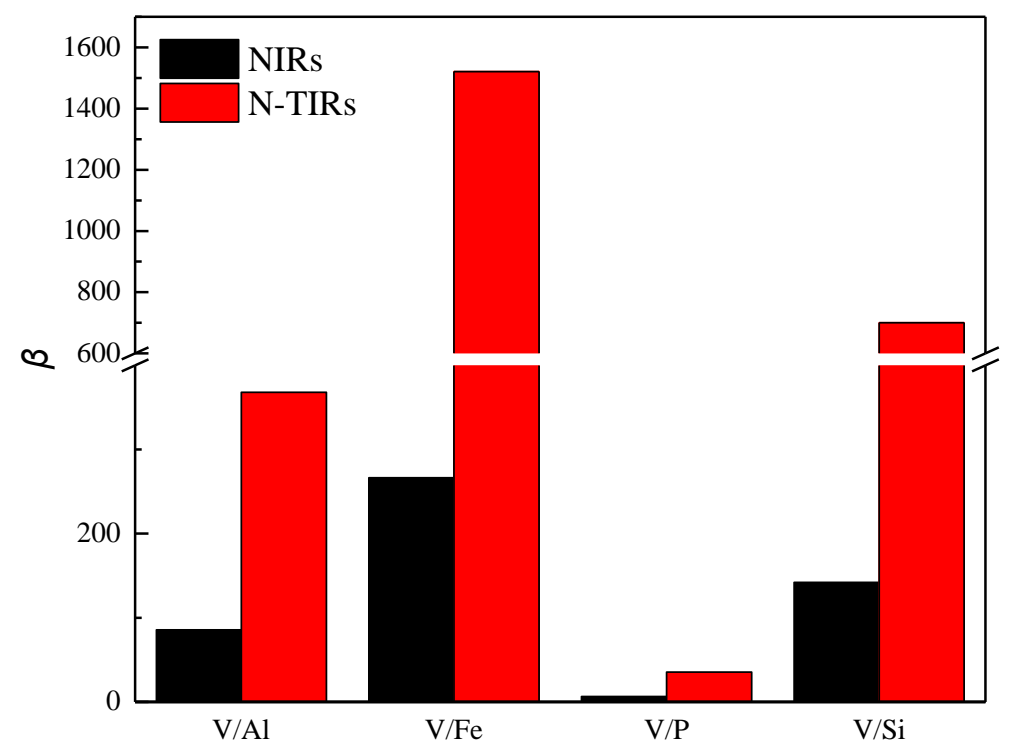

Figure 11. The separation factor for $V(V)$ over impurities onto N-TIRs and NIRs.

\subsection{Stability of the SIRs}

The cyclic adsorption-desorption experiments of the N-TIRs and NIRs were carried out so as to investigate the stability of the SIRs during cyclic use. Figure 12 reveals that the adsorption capacity of N-TIRs and NIRs for $\mathrm{V}(\mathrm{V})$ both decline with the increasing of the cyclic times. Nevertheless, the decrement in the adsorption capacity of NIRs is more obvious than that of N-TIRs. As is depicted in Figure 12, the adsorption capacity of N-TIRs for $\mathrm{V}(\mathrm{V})$ is $91.50 \%$ of the initial value after the ninth cyclic adsorption in comparison to that $77.83 \%$ for the NIRs. As mentioned above, the extractants going deeper into the pores of the N-TIRs is difficult to lose from the pores of N-TIRs when compared to that impregnated in the shallower pores of NIRs [20]. Thus, the stability of N-TIRs is stronger than that of NIRs.

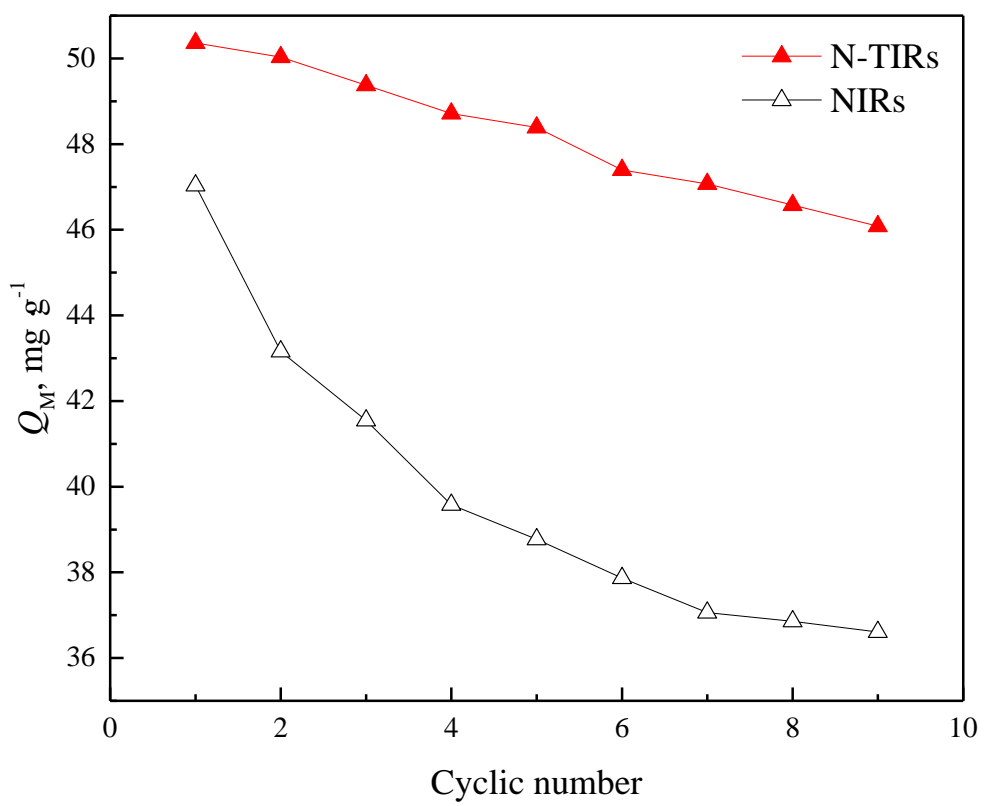

Figure 12. Stability of the N-TIRs and NIRs during cyclic use. 


\section{Conclusions}

N-TIRs were prepared so as to investigate the effects of the addition of TBP on the preparation process of NIRs and the adsorption performance of such prepared SIRs for V(V). The significant characteristics of the N-TIRs are obtained, as follows:

(1) The addition of TBP can accelerate the impregnation rate and improve the impregnation ratio of N235 onto the macroporous resins. The impregnation equilibrium time of N-TIRs can be significantly shortened to $5 \mathrm{~min}$ in comparison to that of NIRs (240 $\mathrm{min}$ ) and the impregnation ratio of N-TIRs $(57.73 \%)$ is higher than that of the NIRs $(36.95 \%)$.

(2) FTIR analyses demonstrate that N235 can interact with TBP during the impregnation process, resulting in the improvement of the extractability of N235, which enhances the adsorption capacity and the separation capability of the N-TIRs for $V(V)$ when compared with that of the NIRs $\left(50.95 \mathrm{mg} \cdot \mathrm{g}^{-1}\right.$ versus $\left.46.73 \mathrm{mg} \cdot \mathrm{g}^{-1}\right)$. It is confirmed by SEM-EDS analyses that more extractants can enter deeper into the pores of the resins with the addition of TBP, thus the stability of the N-TIRs is higher than that of the NIRs during the cyclic use.

(3) The kinetics results show that the pseudo-second order model fits better for the adsorption process of $\mathrm{V}(\mathrm{V})$ onto the N-TIRs and NIRs, and the chemical reaction is the rate-limiting step of the adsorption process of $\mathrm{V}(\mathrm{V})$. The reaction constant $\left(K_{\mathrm{s}}\right)$ implies that the reaction rate of $\mathrm{V}(\mathrm{V})$ with extractants impregnated onto N-TIRs is faster than that onto NIRs, which results in the shorter adsorption equilibrium time of the NTIRs $(6 \mathrm{~h})$ when compared to NIRs (10 h).

In sum, the addition of TBP can impose many positive effects on the preparation of NIRs and adsorption performance for $\mathrm{V}(\mathrm{V})$, such as accelerating the impregnation rate and improving the impregnation ratio of the extractants onto the NIRs, obviously shortening the adsorption equilibrium time of the NIRs and enhancing the adsorption capacity and stability of the NIRs. This study may propose a new sight on the preparation of SIRs by adding a secondary reagent to improve the performance of SIRs.

Author Contributions: S.B., B.C. and Y.Z. conceived and designed the experiments; B.C. and R.Z. performed the experiments; B.C., S.B. and R.Z. analyzed the data; S.B. and Y.Z. contributed reagents/materials/analysis tools; B.C. and S.B. wrote the paper.

Funding: This research was supported by the Major Technical Innovation Project of Hubei Province (2018ACA157), National Key Science-Technology Support Programs of China (2015BAB03B05) and the Excellent Dissertation Cultivation Funds of WUT (2017-YS-054).

Conflicts of Interest: There are no conflicts to declare.

\section{References}

1. Saitoh, T.; Suzuki, S.; Hiraide, M. Solid phase extraction of some precious metals from hydrochloric acid to polystyrene-divinylbenzene porous resin impregnated with polyoxyethylene-type nonionic surfactant. J. Chromatogr. A 2005, 1097, 179-182. [CrossRef] [PubMed]

2. Lee, G.S.; Uchikoshi, M.; Mimura, K.; Isshiki, M. Separation of major impurities Ce, Pr, Nd, Sm, Al, Ca, Fe, and Zn from La using bis(2-ethylhexyl) phosphoric acid (D2EHPA)-impregnated resin in a hydrochloric acid medium. Sep. Purif. Technol. 2010, 71, 186-191. [CrossRef]

3. Metwally, S.S.; Hassan, M.A.; Aglan, R.F. Extraction of copper from ammoniacal solution using impregnated amberlite XAD-7 resin loaded with LIX-54. J. Environ. Chem. Eng. 2013, 1, 252-259. [CrossRef]

4. Babić, K.; Ham, L.V.D.; Haan, A.D. Recovery of benzaldehyde from aqueous streams using extractant impregnated resins. React. Funct. Polym. 2006, 66, 1494-1505. [CrossRef]

5. Burghoff, B.; Schiferli, J.; Marques, J.S.; Haan, A.B.D. Extractant screening and selection for methyl tert-butyl ether removal from aqueous streams. Chem. Eng. Sci. 2009, 64, 2887-2892. [CrossRef]

6. Liang, L.; Bao, S.; Zhang, Y.; Tang, Y. Separation and recovery of V(IV) from sulfuric acid solutions containing $\mathrm{Fe}(\mathrm{III})$ and $\mathrm{Al}(\mathrm{III})$ using bis(2-ethylhexyl)phosphoric acid impregnated resin. Chem. Eng. Res. Des. 2016, 111, 109-116. [CrossRef] 
7. Tang, Y.; Bao, S.; Zhang, Y.; Liang, L. Effect of support properties on preparation process and adsorption performances of solvent impregnated resins. React. Funct. Polym. 2017, 113, 50-57. [CrossRef]

8. Warshawsky, A. South African Patent 71/5637, 1971.

9. Grinstead, R. On Contract NO. 14-12-808 to the Water Quality Office of US Environmental Protection Administration; Reported by the Dow Chemical Co.: Midland, Michigan, USA, January 1971.

10. Kroebel, R.; Meyer, A. West German Patent 2,162,951, 1971.

11. Bao, S.; Tang, Y.; Zhang, Y.; Liang, L. Recovery and Separation of Metal Ions from Aqueous Solutions by Solvent-Impregnated Resins. Chem. Eng. Technol. 2016, 39, 1377-1392. [CrossRef]

12. Navarro, R.; Saucedo, I.; Núñez, A.; Ávila, M.; Guibal, E. Cadmium extraction from hydrochloric acid solutions using Amberlite XAD-7 impregnated with Cyanex 921 (tri-octyl phosphine oxide). React. Funct. Polym. 2008, 68, 557-571. [CrossRef]

13. Ma, Y.; Wang, X.; Wang, M.; Jiang, C.; Xiang, X.; Zhang, X. Separation of V(IV) and Fe(III) from the acid leach solution of stone coal by D2EHPA/TBP. Hydrometallurgy 2015, 153, 38-45. [CrossRef]

14. Cheraghi, A.; Ardakani, M.S.; Alamdari, E.K.; Fatmesari, D.H.; Darvishi, D.; Sadrnezhaad, S.K. Thermodynamics of vanadium (V) solvent extraction by mixture of D2EHPA and TBP. Int. J. Miner. Process. 2015, 138, 49-54. [CrossRef]

15. Li, X.; Deng, Z.; Wei, C.; Li, C.; Li, M.; Fan, G.; Huang, H. Solvent extraction of vanadium from a stone coal acidic leach solution using D2EHPA/TBP: Continuous testing. Hydrometallurgy 2015, 154, 40-46. [CrossRef]

16. Alamdari, E.K.; Darvishi, D.; Sadrnezhaad, S.K.; Haghshenas, D.F. Synergistic effect of TBP on separation of zinc and cadmium with D2EHPA. In Proceedings of the International Solvent Extraction Conference (ISEC), Beijing, China, 19-23 September 2005.

17. Zheng, R.; Bao, S.; Zhang, Y.; Chen, B. Synthesis of Di-(2-ethylhexyl) phosphoric acid (D2EHPA)-Tributyl phosphate) (TBP) Impregnated Resin and Application in Adsorption of Vanadium(IV). Minerals 2018, 8, 206. [CrossRef]

18. Chen, B.; Bao, S.; Zhang, Y. Preparation of N235-impregnated resin and its adsorption for vanadium. Chin. J. Rare Met. 2017, 42, 891-896.

19. Shen, C.; Zhang, Y.; Bao, S.; Huang, J.; Yang, X. Separation and enrichment of vanadium from stone coal acidic leach solution using tertiary amine N235. Chin. J. Rare. Met. 2017, 41, 422-428.

20. Chen, B.; Bao, S.; Zhang, Y.; Zheng, R. Ultrasound-assisted synthesis of N235-impregnated resins for vanadium (V) adsorption. R. Soc. Open Sci. 2018, 5, 171746. [CrossRef] [PubMed]

21. Li, X.; Wei, C.; Deng, Z.; Li, M.; Li, C.; Fan, G. Selective solvent extraction of vanadium over iron from a stone coal/black shale acid leach solution by D2EHPA/TBP. Hydrometallurgy 2011, 105, 359-363. [CrossRef]

22. Baaden, M.; Burgard, M.; Wipff, A.G. TBP at the water-oil interface: The effect of TBP concentration and water acidity investigated by molecular dynamics simulations. J. Phys. Chem. B 2001, 105, 11131-11141. [CrossRef]

23. Fatmehsari, D.H.; Darvishi, D.; Etemadi, S.; Hollagh, A.R.E.; Alamdari, E.K.; Salardini, A.A. Interaction between TBP and D2EHPA during $\mathrm{Zn}, \mathrm{Cd}, \mathrm{Mn}, \mathrm{Cu}, \mathrm{Co}$ and Ni solvent extraction: A thermodynamic and empirical approach. Hydrometallurgy 2009, 98, 143-147. [CrossRef]

24. Nguyen, N.V.; Lee, J.C.; Jeong, J.; Pandey, B.D. Enhancing the adsorption of chromium(VI) from the acidic chloride media using solvent impregnated resin (SIR). Chem. Eng. J. 2013, 219, 174-182. [CrossRef]

25. Nguyen, N.V.; Lee, J.; Jha, M.K.; Yoo, K.; Jeong, J. Copper recovery from low concentration waste solution using Dowex G-26 resin. Hydrometallurgy. 2009, 97, 237-242. [CrossRef]

26. Ho, Y.S.; Mckay, G. The kinetics of sorption of divalent metal ions onto sphagnum moss peat. Water. Res. 2000, 34, 735-742. [CrossRef]

27. Cortina, J.L.; Miralles, N. Kinetic studies on heavy metal ions removal by impregnated resins containing di-(2,4,4-trymethylpentyl) phosphinic acid. Solvent. Extr. Ion Exc. 1997, 15, 1067-1083. [CrossRef]

(C) 2018 by the authors. Licensee MDPI, Basel, Switzerland. This article is an open access article distributed under the terms and conditions of the Creative Commons Attribution (CC BY) license (http://creativecommons.org/licenses/by/4.0/). 